\title{
Genetic polymorphism in the asporin gene is not a key risk factor for osteoarthritis: Evidence based on an updated cumulative meta-analysis
}

\author{
JING WANG ${ }^{1}$, AIMIN YANG ${ }^{2}$, JIE ZHANG $^{2}$, NA SUN $^{1}$, XIANGWEN LI $^{1}$, XINGHUI LI $^{1}$, \\ QILING LIU ${ }^{1}$, JUN LI $^{3}$, XIAOMEI REN ${ }^{1}$, ZUNHUA KE $^{1}$ and RONGQIANG ZHANG ${ }^{1}$ \\ ${ }^{1}$ School of Public Health, Shaanxi University of Chinese Medicine, Xi'an, Shaanxi 712046, P.R. China; \\ ${ }^{2}$ School of Public Health, Brown University, Providence, RI 02906, USA; ${ }^{3}$ Department of School Health, \\ Center for Disease Control and Prevention of Xi'an, Xi'an, Shaanxi 710054, P.R. China
}

Received September 29, 2017; Accepted February 12, 2018

DOI: $10.3892 /$ etm.2018.5888

\begin{abstract}
To provide an evidence-based medical basis for the treatment of osteoarthritis, a meta-analysis was performed to assess the association between asporin (ASPN) gene polymorphism and susceptibility to osteoarthritis (OA). The current study searched the literature from January 1st, 1915 through February 1st, 2017 using the Cochrane Library, PubMed, the Excerpta Medica database (EMBASE) and three main Chinese databases (VIP, CNKI and Wan Fang). Cohort and case-control studies that explored the association between different types of ASPN alleles and OA susceptibility were evaluated. The K/L grading system, clinical and radiological diagnoses were used for OA diagnosis. A random-effects model was used in a pooled analysis to adjust for heterogeneity of the included studies, and the differences between treatment groups were reported as odds ratio (OR), 95\% confidence intervals (CIs) and P-values. Begg's funnel plots and Egger's tests were used to assess publication bias in the present meta-analysis. Following document retrieval and screening, a total of 10 studies were deemed eligible, including 4,842 patients and 3,661 healthy subjects. Results of the multivariate meta-regression analysis revealed that the study sample size was a source of heterogeneity between studies. The D17 allele was a risk factor for the development of $\mathrm{OA}(\mathrm{OR}=1.33,95 \% \mathrm{CI}: 1.02-1.73, \mathrm{P}<0.05)$. The other alleles were not considered as risk factors for development of $\mathrm{OA}(\mathrm{P}>0.05)$. The results of the meta-analysis verified that
\end{abstract}

Correspondence to: Dr Rongqiang Zhang, School of Public Health, Shaanxi University of Chinese Medicine, Shiji Avenue, Xi'an-Xianyang New Ecomic Zone, Xi'an, Shaanxi 712046, P.R. China

E-mail: zhangrqxianyang@163.com

Key words: osteoarthritis, susceptibility, asporin, polymorphism, meta-analysis
ASPN polymorphisms were not significantly relevant to an increased OA risk. However, the mechanisms contributing to the association between ASPN polymorphisms and OA risk still require further study.

\section{Introduction}

Osteoarthritis (OA) is a common disease of the skeletal system. As the most frequent form of joint disease, OA can occur in any joint but primarily affects knees, hips, hands and the spine, thus constituting a leading cause of musculoskeletal disability worldwide $(1,2)$. Degradation of hyaline articular cartilage and remodeling of the subchondral bone with sclerosis are the main features of OA $(3,4)$. At first, patients with OA present with joint stiffness and soreness (5). The articulation may show some swelling caused by an effusion and synovitis. When the bare bony surfaces grate against each other, locking may also occur, which will cause more serious pain (6) and will result in symptoms that decrease quality of life. A previous epidemiological survey showed that approximately $10 \%$ of the world's population aged over 60 years may present with symptomatic OA $(7,8)$. Moreover, according to the World Health Organization (WHO), OA has been the fourth major cause of disability for many years, with an incidence rate of 40\% in the 55-64 age group (9). As the global population mages, the incidence of OA increases year after year. In clinical practice, diagnosis of OA is based mainly on radiological examination and clinical assessment, and a key point for OA diagnosis is to identify early stage onset and progression (10). Additionally, OA is considered to be a polygenic and multifactorial illness (11-13). Studies have shown that age, sex, obesity, genetics, ethnicity, behavioral influences, occupation and environmental factors, and particularly genetic factors strongly affect the occurrence and development of OA $(14,15)$. Exploring the genetic factor mechanism would prove helpful in the early diagnosis of OA.

The asporin (ASPN) gene has been reported as a predisposing gene for $\mathrm{OA}$ in several previous studies and encodes a cartilage extracellular protein belonging to the small leucine-rich proteoglycan (SLRP) family (3). These 
proteins can regulate chondrogenesis by binding to cartilage transforming growth factor- $\beta$ (TGF- $\beta$ ) and inhibiting the expression of the TGF- $\beta 1$-induced gene in cartilage (16-18). In normal cartilage, ASPN is expressed at low levels but in OA articular cartilage, it is abundantly expressed, thus playing a key role in cartilage metabolism (19). The D14 allele of ASPN has been reported as a gene potentially contributing to knee OA (KOA), and there is a significant difference in the allele frequency of ASPN D13 between female OA patients and controls (20). A number of studies have examined the association between ASPN polymorphisms and OA, but their results and conclusions have been contradictory. Therefore, there is not yet strong evidence of the association between OA and ASPN. Therefore, it is necessary to conduct additional studies to confirm the relationship between OA and ASPN. In the present study, an updated, more comprehensive and more detailed cumulative meta-analysis was performed based on the newly published original studies evaluating the association between ASPN polymorphism and OA susceptibility, to investigate whether the D-repeat polymorphism is associated with susceptibility to OA and whether it can provide a reference for the early diagnosis of OA.

\section{Materials and methods}

Search strategy. To perform this meta-analysis, the Cochrane Library, PubMed and Excerpta Medica databases (EMBASE) were used to comprehensively search the relevant literature. The following keywords were used: '(Asporin or ASPN), (Osteoarthritis or OA), and (Asporin and ASPN or Osteoarthritis and OA)'. In addition, the Chinese electronic databases: VIP, CNKI and Wan Fang were used to search the relevant literature. All articles that were selected for analysis focused on ASPN gene polymorphisms and OA susceptibility and had been published from January 1st, 1915 through February 1st, 2017. By using the aforementioned search strategy, two authors independently completed screening the records and then assessed the final results. The study selection flowchart is shown in Fig. 1.

Inclusion and exclusion criteria. To be included in the meta-analysis, studies had to meet the following criteria: i) They had to be a case-control study (or cohort study); ii) studies had to provide data on the number of studies and ratios, and OA diagnosis had to be performed using a standardized method; and iii) they had to contain a study of genotype and the ASPN allele, the relevant odds ratio (OR) and the 95\% confidence interval (CI). Exclusion criteria were: i) Literature review or incomplete data; ii) repeated published documents and overlapping data; and iii) disease and gene research limited to one family. There were no restrictions on the country and language under study.

Data extraction. Two reviewers (Jing Wang and Rongqiang Zhang) independently reviewed and extracted data from all qualified studies. Data extraction from original studies included first author's name, year of publication, total sample size and age of OA patients and controls, diagnostic criteria, country where the study was performed, ethnicity of the participants, OA sites and genotype frequencies of the two groups. The continent of origin was categorized as follows: Europe or America $(=1)$ and Asia (=2). Total sample sizes of OA patients and controls were categorized as $<1,000(=1)$ and $\geq 1,000(=2)$. Moreover, OA sites were categorized as $\mathrm{KOA}(=1)$ and hand or hip OA $(=2)$. The two investigators carefully checked the data extraction information and reached consensus to ensure accuracy of the extracted data. In the case of disagreement, the two reviewers double-checked the original data together and then discussed them in order to reach an agreement. If they failed to reach an agreement, a third reviewer (Aimin Yang) was invited to the discussion.

Methodological quality assessment. The methodological quality of the included studies was independently assessed by two reviewers (Jing Wang and Rongqiang Zhang), according to our revised Scale for Quality Assessment (Table I) based on the scales of Thakkinstian et al (21) and Qin et al (22). The revised scale covered criteria such as representativeness of cases, credibility of controls, ascertainment of OA, genotyping examination, Hardy-Weinberg equilibrium (HWE) and association assessment. Any disagreement related to methodological quality was resolved by consensus. Scores ranged from 0 to 12 . Articles that were assigned a score $<8$ were considered 'low-quality' studies, whereas papers with a score $\geq 8$ were considered 'high-quality' studies.

Statistical analysis. RevMan software v5.2 (The Cochrane Collaboration, Oxford, UK) was used to perform the metaanalysis. When the selected original studies met the criteria for homogeneity $\left(I^{2}<50 \%\right.$ or $\left.\mathrm{P}>0.05\right)$, a fixed-effects model was chosen to conduct the meta-analysis. If they met the criteria for heterogeneity $\left(I^{2}>50 \%\right.$ or $\left.\mathrm{P}<0.05\right)$, a random-effects model was chosen to conduct the meta-analysis. Univariate and multivariate meta-regression analyses were then performed to explore the source of heterogeneity between studies using Stata 13.0 software (Stata Corp, College Station, TX, USA). In meta-regression analyses, $\log$ OR (where OR stands for the relevant OR of cases vs. control) was taken as the dependent variable. The sample size, OA sites and continent of origin were taken as independent variables. If necessary, a subgroup analysis was conducted according to the source of heterogeneity. Publication bias was analyzed with a funnel plot and Begg's test.

\section{Results}

Literature search and study selection. A total of 34,084 relevant articles were searched from the Cochrane Library, PubMed, the Excerpta Medica database, VIP, CNKI and Wan Fang. Finally, 10 articles including 11 studies (Kizawa's research included both an independent case-control study and an independent cohort study) were included in our meta-analysis.

Study characteristics. In total, 8,503 participants (4,842 OA patients and 3,661 controls) were eligible for inclusion, involving three different regions, each of which encompassed a distinct human race, and four types of OA pathogenesis 


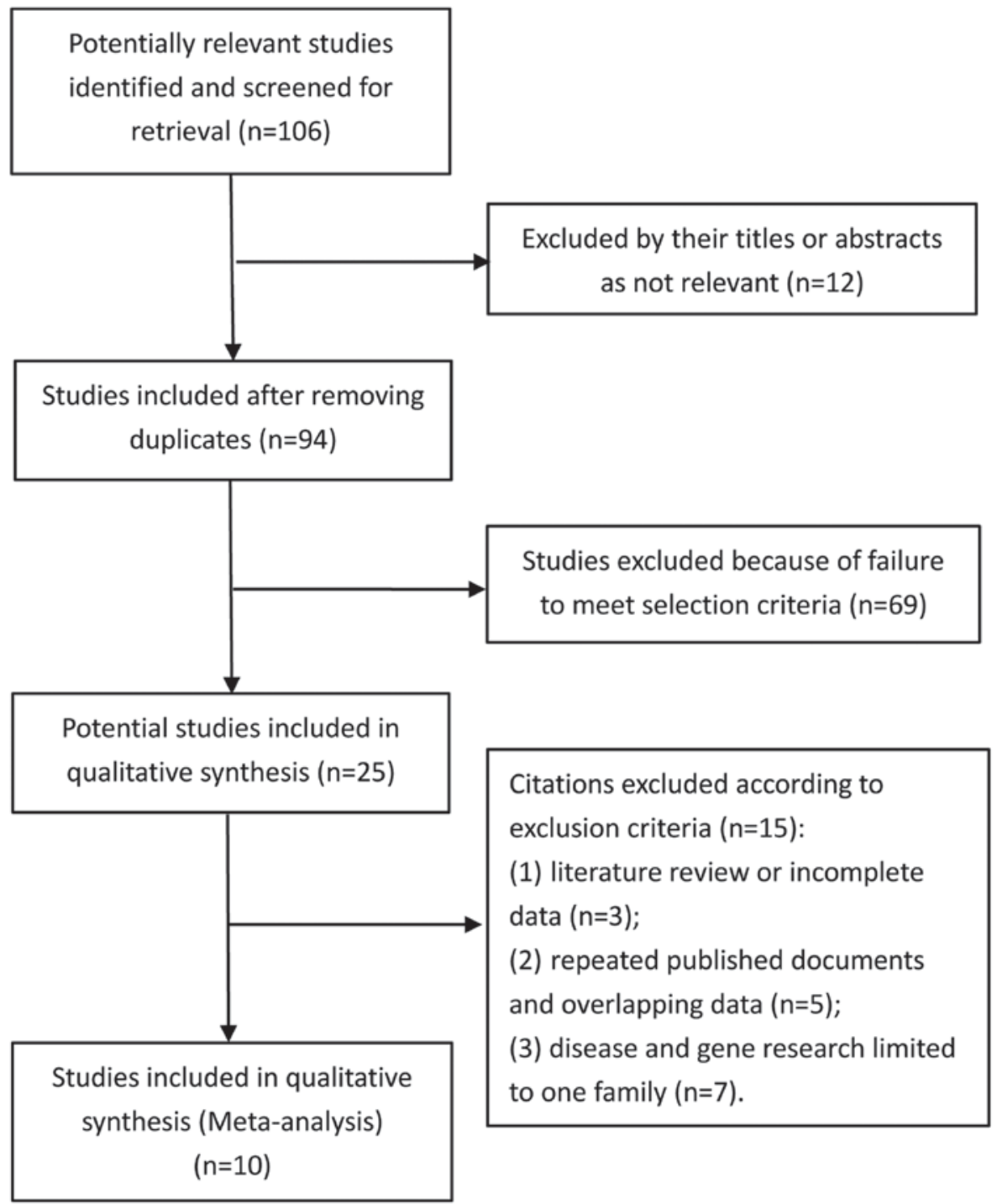

Figure 1. Flowchart representing study selection for the meta-analysis.

research. Cohort studies included 137 patients and 234 controls while case-control studies comprised 4,705 patients and 3,427 controls. A total of 4 articles focused on Asians, 4 on Europeans and 2 on Americans. Among them, 7 studies examined KOA and 4 studies focused on hand or hip OA. Participants included in both case and control groups were all OA patients and healthy controls confirmed by standard diagnostic criteria (K/L grading system ${ }^{5}$, Criteria of American Rheumatology College, clinical and radiologic diagnosis), respectively. Polymorphisms of the ASPN gene were detected using multiplex polymerase chain reaction (PCR) and single nucleotide polymorphism (SNP) analyses, which were performed using the GenomeLab SNPstream Genotyping System (Beckman Coulter, Fullerton, CA, USA). The characteristics of all included studies are presented in Table II.

Methodological quality. Studies were generally of good quality with a mean score of 9.55 (Table II), of which eight studies $(66.67 \%$ ) had a score equal to or greater than 8 , while the three other studies $(33.33 \%)$ received a score lower than 8 . Two-thirds of the studies included in this meta-analysis were thus classified as of high quality.
Results of overall meta-analysis. The association between ASPN polymorphisms and susceptibility to OA is shown in Table III. The results of the analyses of heterogeneity showed that there was heterogeneity on $\mathrm{D} 12$ in seven studies $(\mathrm{P}=0.02$, $\left.I^{2}=62 \%\right)$, on D13 $\left(\mathrm{P}=0.01, I^{2}=55 \%\right)$ and D14 $\left(\mathrm{P}=0.0001, I^{2}=71 \%\right)$ in eleven studies, and on D15 $\left(\mathrm{P}=0.005, I^{2}=63 \%\right)$ in nine studies. Therefore, random-effects models were used for the meta-analysis of D12-D15. There was no significant heterogeneity on either D16 $\left(\mathrm{P}=0.07, I^{2}=47 \%\right)$ or $\mathrm{D} 17\left(\mathrm{P}=0.37, I^{2}=8 \%\right)$ in eight studies, or on $\mathrm{D} 18\left(\mathrm{P}=0.38, I^{2}=6 \%\right)$ in six studies. Consequently, fixed-effects models were used to calculate the pooled ORs of D16, D17 and D18. The pooled OR and its 95\% CI for the D17 allele vs. others combined indicated that D17 alleles were a statistically significant risk factor for OA development (OR=1.33, 95\% CI: 1.02-1.73). No statistically significant association was found between OA and ASPN D12, D13, D14, D15, D16 or D18 alleles.

Test of heterogeneity. Because the included studies showed significant heterogeneity for D12, D13, D14 and D15, univariate and multivariate meta-regression analyses were performed to explore the source of this heterogeneity. For the reason that the 
Table I. Scale for quality assessment.

Representativeness of cases

Selected from population

Selected from any OA/surgery service

Selected without clearly defined sampling frame or with extensive inclusion/exclusion criteria

Credibility of controls

Population-or neighbor-based

Blood donors or volunteers

Hospital-based (Joint diseases-free patients)

Healthy volunteers, but without total description

Not described

Ascertainment of OA

Clinical and X-ray or MRI confirmation

Diagnosis of OA by patient medical record

Not described

Genotyping examination

Genotyping done under 'blinded' condition

Unblinded or not mentioned

Hardy-Weinberg equilibrium

Hardy-Weinberg equilibrium in controls

Hardy-Weinberg disequilibrium in controls

No checking for Hardy-Weinberg disequilibrium

Association assessment

Assess association between genotypes and OA with appropriate statistics and adjustment for confounders

Assess association between genotypes and OA with appropriate statistics without adjustment for confounders

Inappropriate statistics used

OA, osteoarthritis; MRI, magnetic resonance imaging.

number of studies focusing on D12 was only 7 and the frequency of D12 alleles reported in the original studies was small, meta-regression analyses were only conducted on D13, D14 and D15. Meta-regression analyses were conducted according to a random-effects model. Univariate analyses were performed separately for the following variables: sample size, continent of origin and OA site. Therefore results showed that there was no statistically significant association between independent variables (sample size, continent of origin and OA site) and the dependent variable (LogOR) for D13, D14, and D15 (Figs. 2-4). However, judging from adjusted R-squared values, the sample size, continent of origin and OA site could all impact the development of OA to a certain degree (Figs. 2-4). Multivariate analysis results showed that sample size played a role in the association between $\mathrm{D} 13$ and $\mathrm{OA}$ risk ( $\mathrm{P}=0.039$; Table IV).

Subgroup analysis. After meta-regression analysis, we conducted a subgroup analysis according to study sample size. Indeed, when sample size was greater than 1,000 the heterogeneity of the D13 gene decreased and became not statistically significant (both $\mathrm{Ps}=0.08$ ). The results indicated that when the sample size $>1,000$, D17 alleles increased the risk for the development of OA $(\mathrm{OR}=1.46,95 \% \mathrm{CI}$ : 1.03-2.07,
$\mathrm{P}<0.05$; Table V). There was no significant association between the other ASPN polymorphisms (D12, D13, D14, D15, D16, D18) and OA in the other subgroup analyses (Tables VI and VII). According to the score of the included studies, we performed a subgroup analysis with score $\geq 8$ and score $<8$ and the results are presented in Table VIII, which indicated that when study design and quality score $>8$, D17 alleles increased the risk for OA $(\mathrm{OR}=1.45,95 \% \mathrm{CI}$ : 1.04-2.02, $\mathrm{P}<0.05)$.

Sensitivity analysis. To assess the influence of each individual study on the pooled ORs, a sensitivity analysis of sequential removal of individual studies was performed. Results shown in Fig. 5 suggest that no individual study significantly affected the pooled ORs, indicating that our meta-analysis results were stable and reliable. Another sensitivity analysis performed by excluding HWE-violating studies did not perturb the overall results.

Publication bias. Publication bias was evaluated by performing Begg's funnel plots and Egger's tests. Fig. 6 shows that the shapes of the Funnel plots were basically symmetrical and did not reveal obvious evidence of asymmetry. All P-values from 


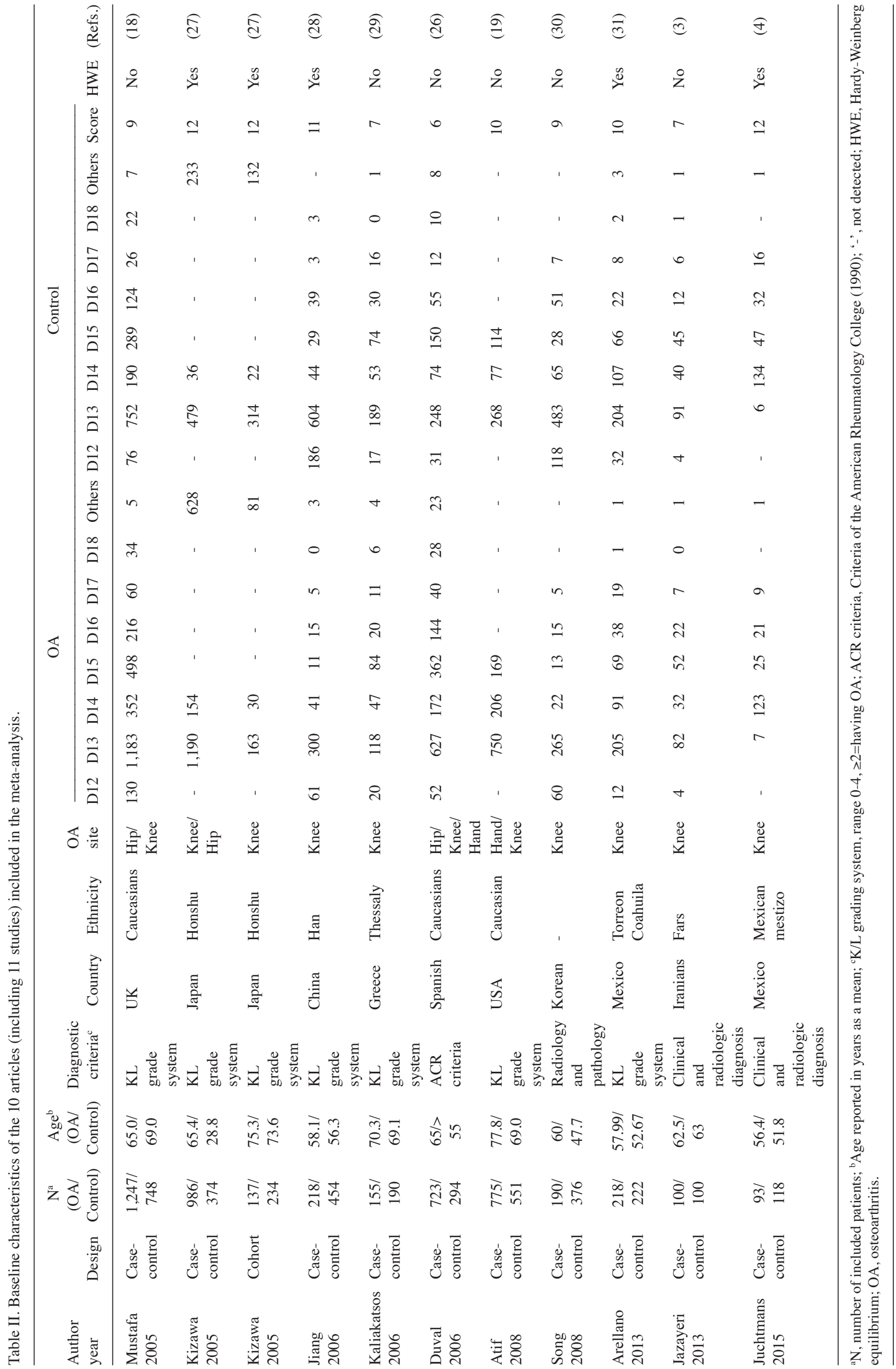


Table III. Summary of ORs and 95\% CIs of the ASPN polymorphisms and OA susceptibility.

\begin{tabular}{|c|c|c|c|c|c|c|c|c|c|}
\hline \multirow[b]{2}{*}{ Comparisons } & \multirow{2}{*}{$\begin{array}{l}\text { No. of } \\
\text { studies }\end{array}$} & \multicolumn{2}{|c|}{ Sample size } & \multicolumn{3}{|c|}{ Test of association } & \multicolumn{3}{|c|}{ Test of heterogeneity } \\
\hline & & $\mathrm{OA}$ & Control & OR & $95 \% \mathrm{CI}$ & $\mathrm{P}$-value & Model $^{\mathrm{a}}$ & $\mathrm{P}$-value & $I^{2}(\%)$ \\
\hline D12 vs. all the others & 7 & 9,684 & 7,322 & 0.81 & $0.61-1.07$ & 0.14 & $\mathrm{R}$ & 0.02 & 62 \\
\hline D13 vs. all the others & 11 & 9,684 & 7,322 & 0.94 & $0.84-1.05$ & 0.25 & $\mathrm{R}$ & 0.01 & 55 \\
\hline D14 vs. all the others & 11 & 9,684 & 7,322 & 1.15 & $0.94-1.40$ & 0.17 & $\mathrm{R}$ & 0.0001 & 71 \\
\hline D15 vs. all the others & 9 & 9,684 & 7,322 & 0.97 & $0.70-1.15$ & 0.71 & $\mathrm{R}$ & 0.005 & 63 \\
\hline D16 vs. all the others & 8 & 9,684 & 7,322 & 1.03 & $0.89-1.19$ & 0.72 & F & 0.07 & 47 \\
\hline D17 vs. all the others & 8 & 9,684 & 7,322 & 1.33 & $1.02-1.73$ & 0.04 & $\mathrm{~F}$ & 0.37 & 8 \\
\hline D18 vs. all the others & 6 & 9,684 & 7,322 & 1.07 & $0.72-1.59$ & 0.75 & $\mathrm{~F}$ & 0.38 & 6 \\
\hline
\end{tabular}

${ }^{a}$ R, Random-effects model; F, Fixed-effects model. OR, odds ratio; CI, confidence interval; ASPN, asporin; OA, osteoarthritis.
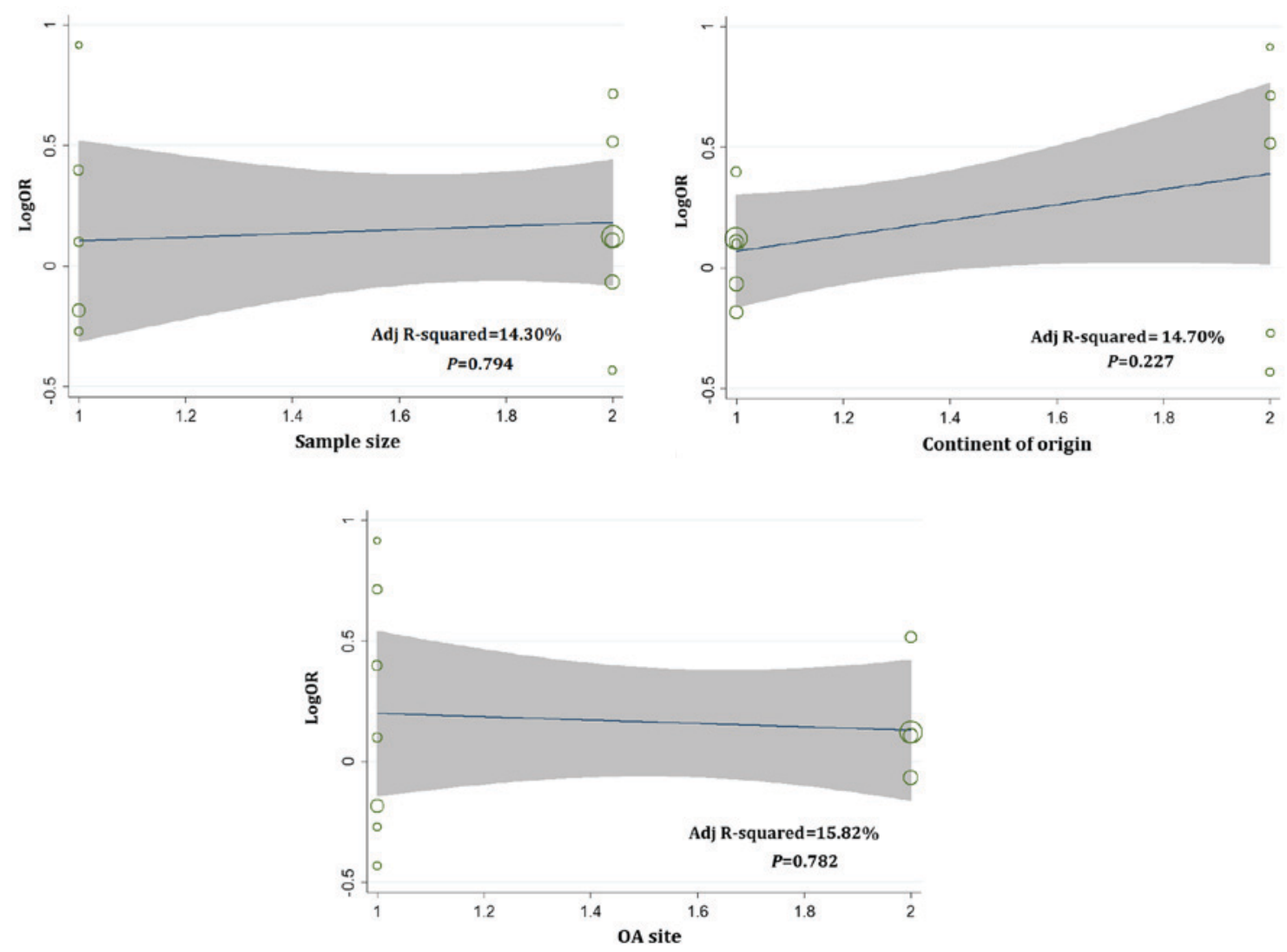

Figure 2. Univariate meta-regression analysis of the association between D13 and the risk for OA. Dot size indicates the weight of observed effect sizes. Error bars indicate $95 \%$ confidence intervals of the regression line. The univariate meta-regression analysis contains studies of different sample size, continent of origin and OA site. OA, osteoarthritis.

Egger's and Begg's tests were greater than 0.05. Therefore, our results suggest no evidence of any publication bias in this meta-analysis.

\section{Discussion}

In the last few years, a greater number of researchers have paid attention to the link between the ASPN gene and OA. The ASPN gene encodes for a protein that is part of the $95 \%$ CI family (23). This ASPN gene is abundantly expressed in human articular cartilage with OA. In the present meta-analysis, we included ten articles (eleven studies) after reviewing the literature for the association between ASPN D-repeat polymorphisms and OA susceptibility (6). In the present study, we aimed to provide more convincing evidence regarding the role of ASPN D-repeat polymorphisms in the incidence of OA.

According to a widely accepted viewpoint, ASPN is a newly identified extracellular matrix protein that contains an $\mathrm{N}$-terminal unique aspartic acid repeat that ranges in length from 8 to 19 residues. The human ASPN gene has 8 

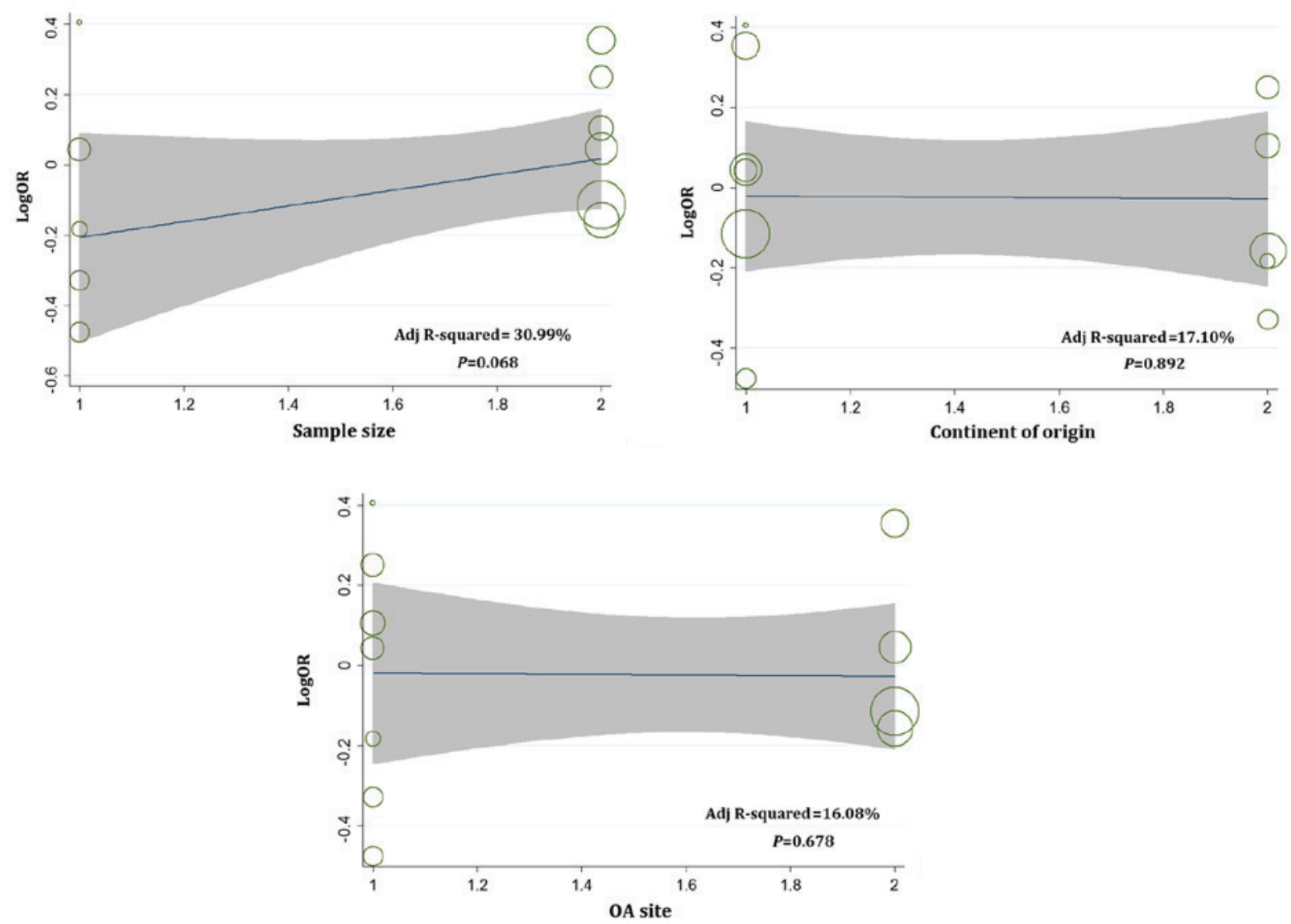

Figure 3. Univariate meta-regression analysis of the association between D14 and the risk of OA. Dot size indicates the weight of the observed effect sizes. Error bars indicate $95 \%$ confidence intervals of the regression line. The univariate meta-regression analysis contains studies of different sample size, continent of origin and OA site. OA, osteoarthritis.
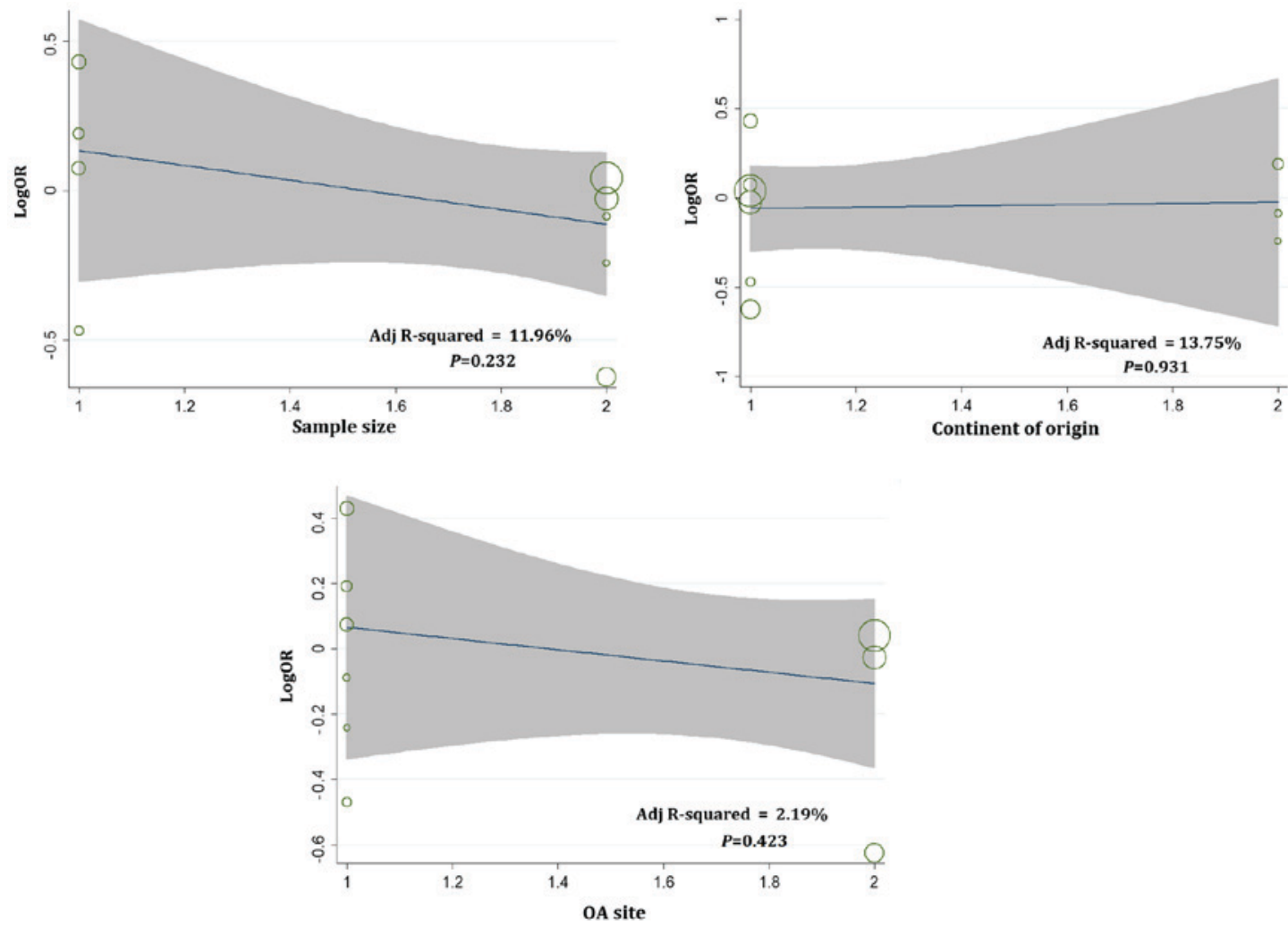

Figure 4. Univariate meta-regression analysis of the association between D15 and the risk of OA. Dot size indicates the weight of the observed effect sizes. Error bars indicate $95 \%$ confidence intervals of the regression line. The univariate meta-regression analysis contains studies of different sample size, continent of origin and OA site. OA, osteoarthritis. 
Table IV. Multivariate meta-regression analysis of the association between D13, D14, D15 and the risk of OA.

\begin{tabular}{lcccrrr}
\hline Genotype & Dependent variables $^{\mathrm{a}}$ & OR & SE & t & P-value & 95\% CI \\
\hline D13 & Continent of origin & 0.8417473 & 0.1296135 & -1.12 & 0.296 & $0.5901624,1.200582$ \\
& OA site & 0.7689406 & 0.1452108 & -1.39 & 0.202 & $0.4974702,1.188553$ \\
& $\mathrm{~N}$ & 1.576952 & 0.290733 & 2.47 & 0.039 & $1.030815,2.412438$ \\
D14 & Continent of origin & 1.366192 & 0.4471786 & 0.95 & 0.368 & $0.6422559,2.906132$ \\
& OA site & 1.054285 & 0.4480117 & 0.12 & 0.904 & $0.3957158,2.808876$ \\
D15 & $\mathrm{N}$ & 0.9812867 & 0.3917458 & -0.05 & 0.963 & $0.3908288,2.463799$ \\
& Continent of origin & 1.147952 & 0.5037435 & 0.31 & 0.764 & $0.3922823,3.359297$ \\
& OA site & 1.145223 & 0.6270224 & 0.25 & 0.813 & $0.2999584,4.372395$ \\
& $\mathrm{~N}$ & 0.6839221 & 0.3202035 & -0.81 & 0.448 & $0.2175071,2.150502$
\end{tabular}

${ }^{\mathrm{a} C o n t i n e n t}$ of origin, Europe or America=1, Asia=2; OA sites, knee $\mathrm{OA}=1$, hand or hip $\mathrm{OA}=2$; Total sample sizes $(\mathrm{N}),<1,000=1, \geq 1,000=2$. OA, osteoarthritis; OR, odds ratio; SE, standard error; CI, confidence interval.

exons. It spans 26 kilobases and is located in chromosome 9q31.1-32, which belongs to the SLRP family. Previous studies have indicated that TGF- $\beta 1$ plays an important role as a regulator of cellular differentiation, proliferation, apoptosis and migration in bone tissue. ASPN can directly bind to the TGF- $\beta 1$ receptor and thus inhibit the expression of cartilage matrix Agc1 and Col2al genes, which are mediated by TGF- $\beta 1$ (24). The interaction between ASPN and TGF- $\beta 1$ can repress the expression of cartilage matrix genes (25). Based on the above findings, many researchers believe that ASPN may play an important role in the occurrence of joint disease (26), including OA.

Many studies have focused on the association between ASPN polymorphisms and OA, but a consensus has not yet been reached. Mustafa et al (18) found that ASPN polymorphism was not a major influence on OA etiology in Caucasians. However, Kizawa et al (27) conducted a cohort study and a case-control study and reported a significant association between the Japanese population's D-repeat polymorphism and OA. Jiang et al (28) studied 218 patients and 454 age-matched controls from the Han Chinese population, revealing for the first time that the OA susceptibility gene was definitely replicated among different ethnic groups. The frequency of the D14 allele in the Greek population showed no significant difference compared with the other alleles, but the frequency of the D13 allele was significantly lower in KOA patients (29). In contrast, the frequency of the D13 allele was found to be significantly different between female KOA patients and controls compared with other alleles (30). In addition, Arellano et al (31) suggested that polymorphisms of the ASPN gene could influence KOA susceptibility, but they indicated that this association also required more extensive research. By investigating the allelic association of the D-repeat polymorphism with OA, Jazayeri et al (3) suggested that the D15 allele could be considered a risk allele only for the female Iranian population. González-Huerta et al (1) reported that the D14 allele of the ASPN polymorphism had a certain impact on the etiology of primary OA of the knee. Therefore, a more objective and accurate conclusion about the link between ASPN polymorphisms and OA risk is required to provide a robust framework for future studies in the field.

From an epidemiological point of view, a case-control study is retrospective and is a causal study allowing exploration of the etiology of diseases. However, a cohort study is more effective than a case-control study for investigation of disease etiology. We conducted an updated comprehensive analysis based on case-control and cohort studies which focused on ASPN polymorphisms and OA risk. The present meta-analysis included 7 studies $(58.33 \%)$ on KOA, 2 studies (16.67\%) on hand OA, and $3(25.00 \%)$ on hip OA. In these 12 studies, 5 (41.67\%) were conducted in Asian populations, four (33.33\%) in European populations and 2 (16.67\%) in American populations. Results of this meta-analysis showed that the D17 allele was a risk factor for $\mathrm{OA}$ and may increase the incidence of $\mathrm{OA}$ $(\mathrm{OR}=1.33$, 95\% CI: $1.02-1.73, \mathrm{P}<0.05)$, particularly in studies with a large sample size $(\mathrm{N}>1,000)$. Results also showed that the other types of alleles did not play an important role in the development of OA. We addressed the association between OA susceptibility and ASPN D-repeat polymorphisms in populations originating from different continents, and the results remained the same. We did not find any significant association between ASPN polymorphisms and OA susceptibility in the subgroup analyses performed according to the OA site and the continent of origin.

We performed a comprehensive review of the literature and designed strict inclusion and exclusion criteria to obtain valid results. Compared with previous studies, our research was more detailed and in addition we conducted different subgroup and meta-regression analyses. There were, however, several limitations in this meta-analysis (10-15,32): i) Because there were inevitably mixed factors in the study, our group analysis could not completely encompass them; ii) the results of this meta-analysis may be misinterpreted due to the limited number of research studies, the shortage of uniform inclusion criteria and the existence of heterogeneity; iii) there is a lack of clinical variables in the literature such as classification of OA severity, and these variables are likely to be related to ASPN polymorphisms; iv) in the subgroup analyses, the results may lack efficiency due to the limited number of studies; and 


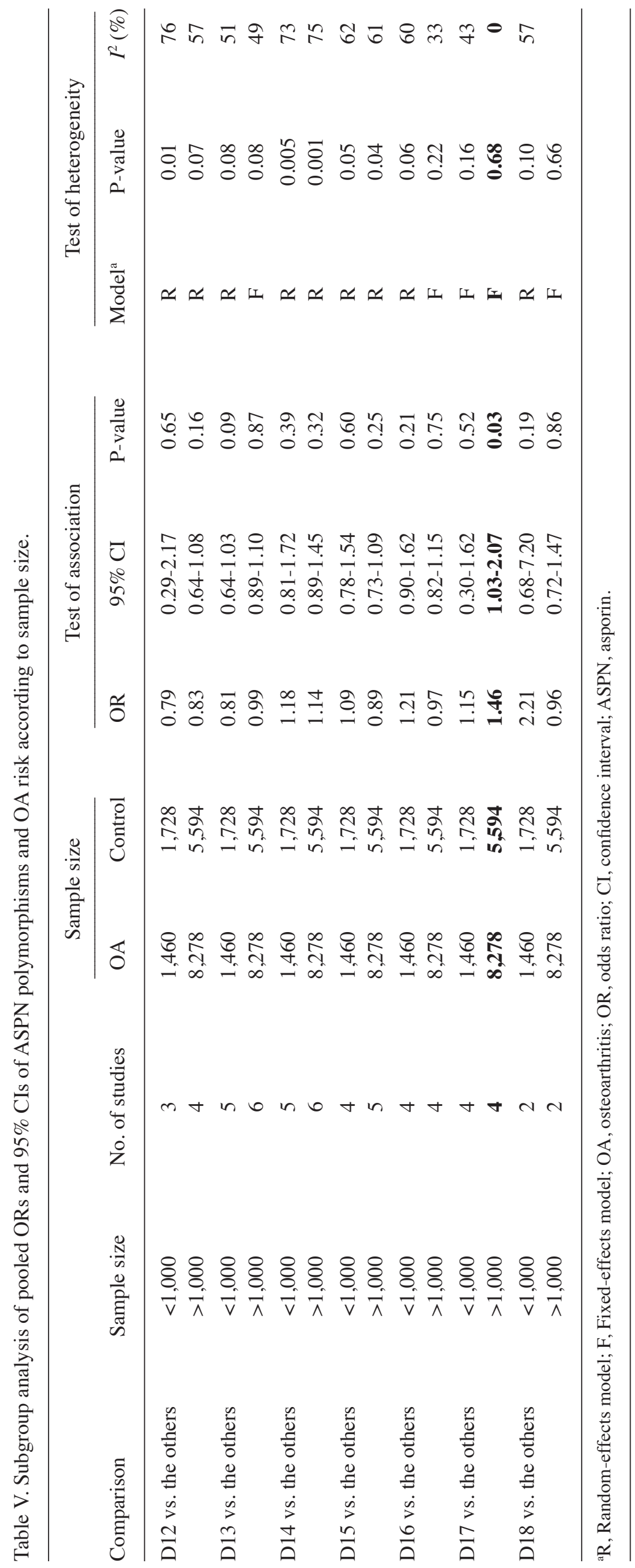




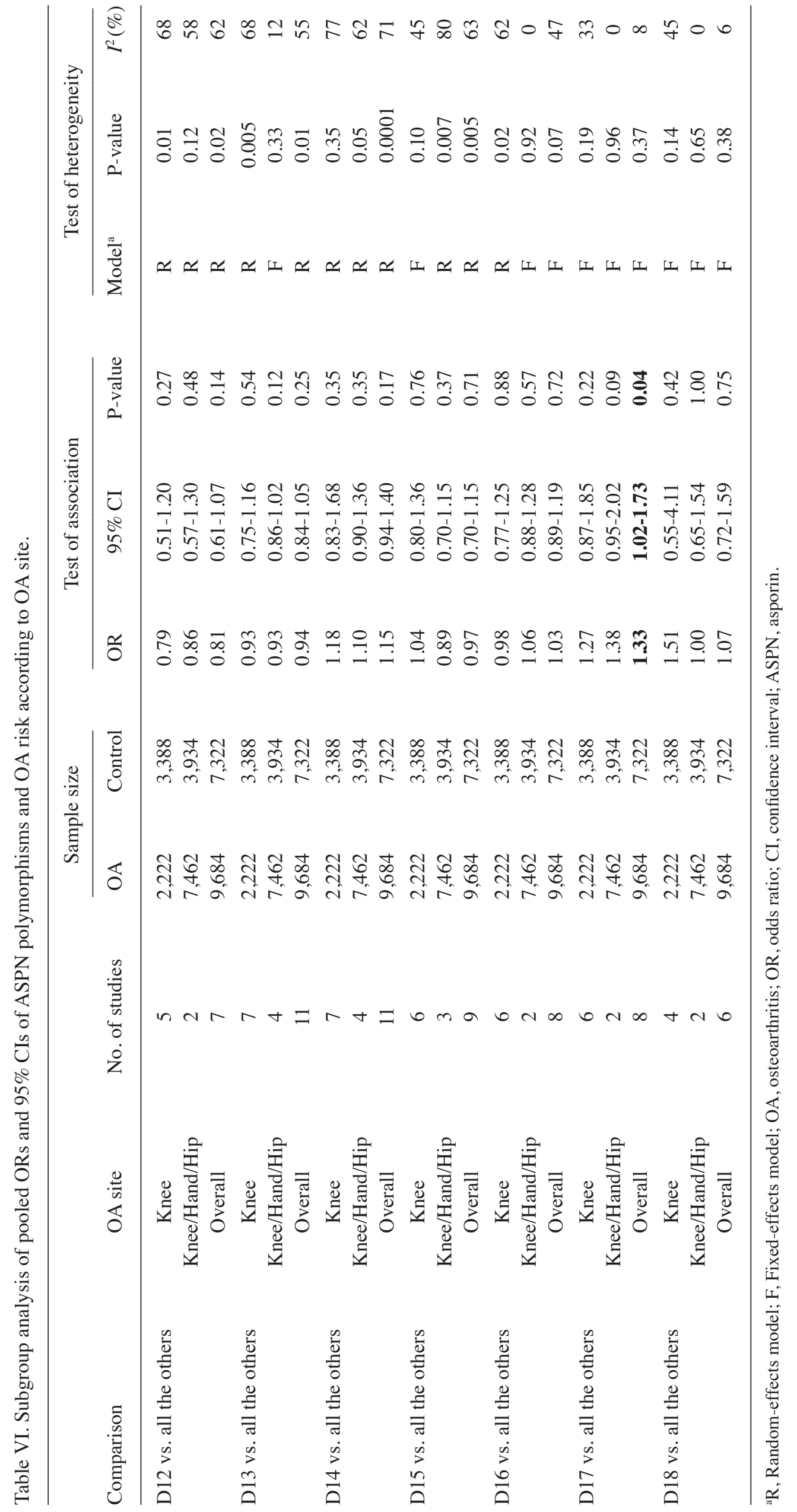




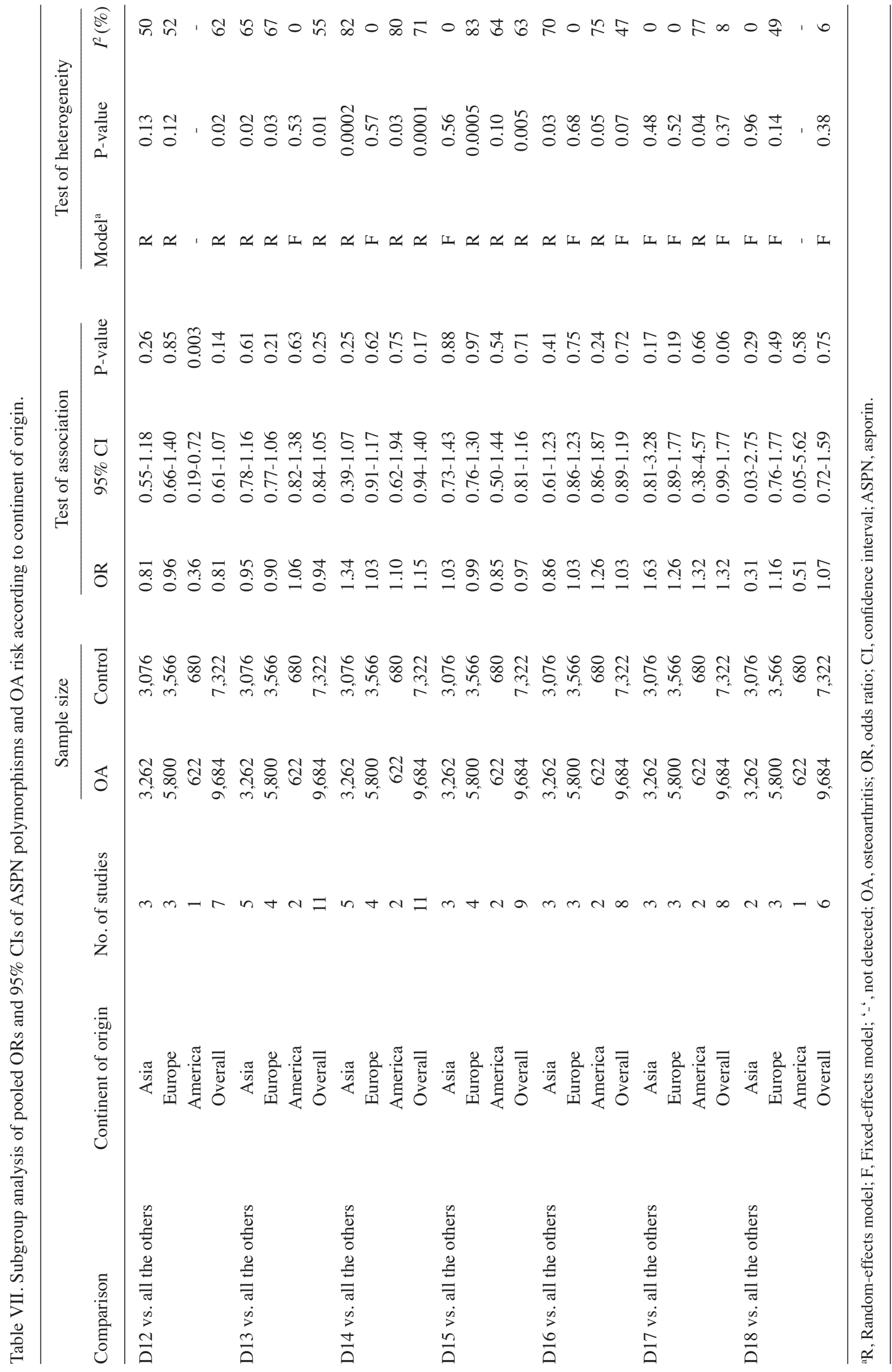




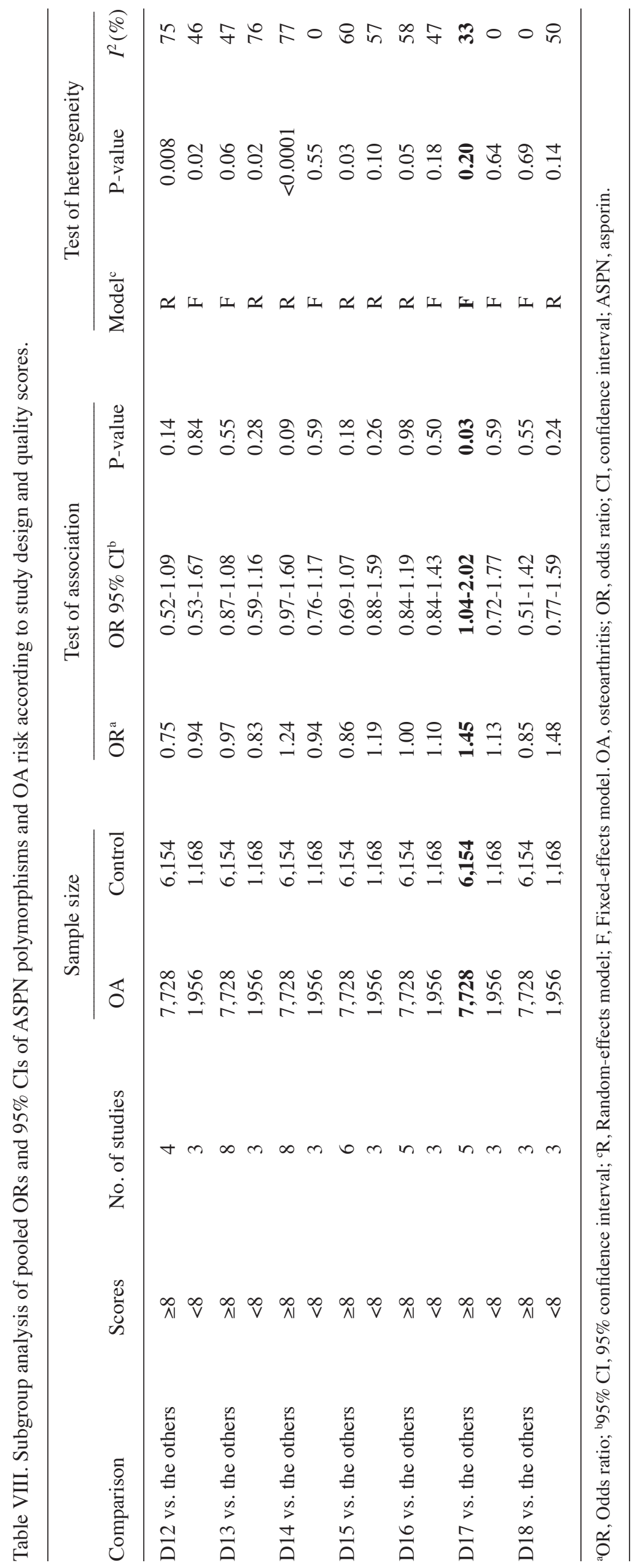



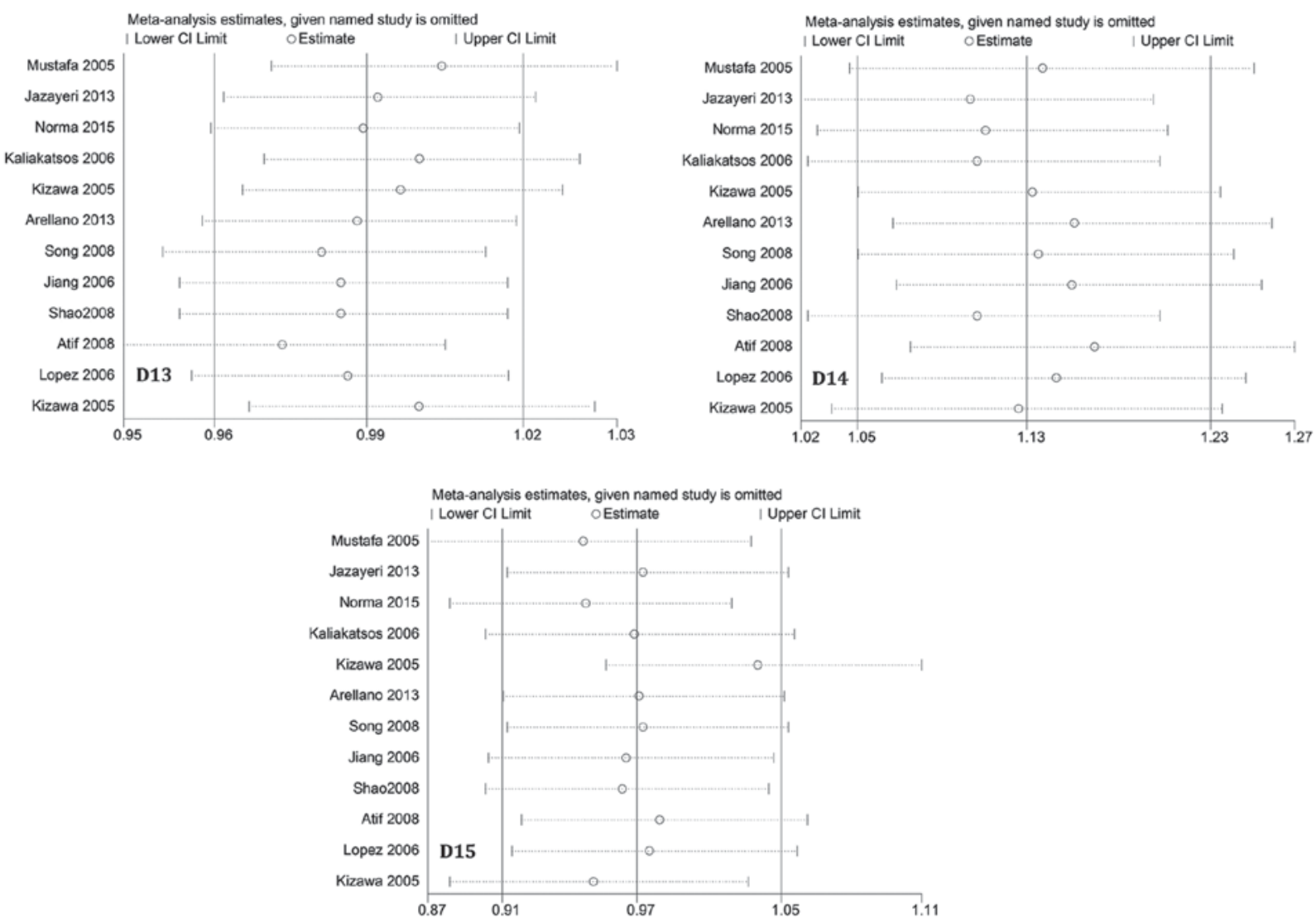

Figure 5. Influence analysis for D13, D14, and D15 alleles vs. the other genotypes in the overall meta-analysis. In the three figures, the middle vertical axis indicates the overall OR and the two vertical axes show 95\% CI of the overall OR. Each hollow circle indicates a pooled OR if the left study is omitted in this meta-analysis. The two ends of every broken line represent the 95\% CI. OR, odds ratio; CI, confidence interval.
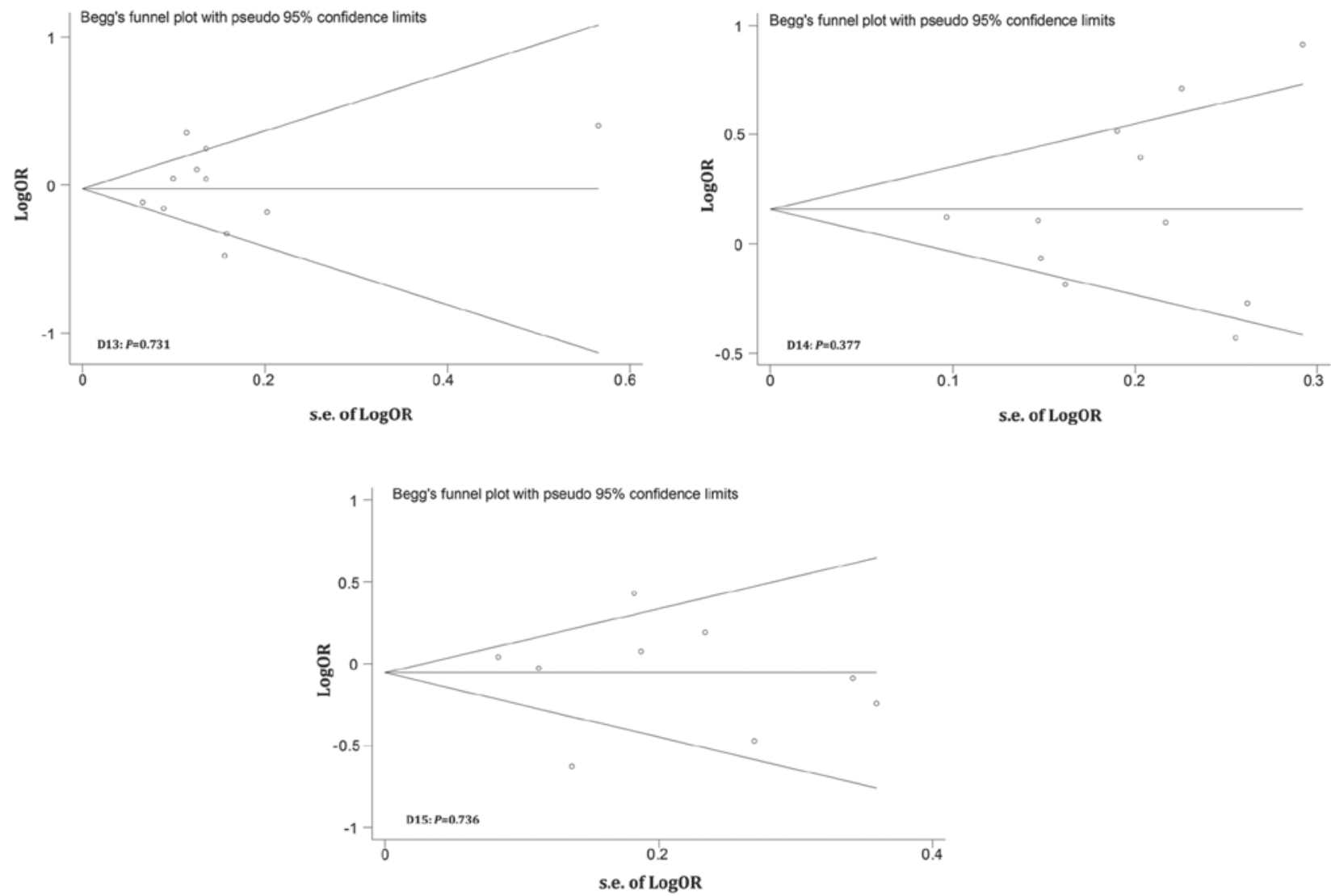

Figure 6. Funnel plots of publication bias of the meta-analysis on the association between ASPN polymorphisms (D13, D14, D15) and OA risk. OA, osteoarthritis. 
v) since the inclusion of the literature may have been biased, even after using a regression test, the inherent bias may have affected the overall analysis. Moreover, as a complex multifactorial disease, development of OA may have been affected by genetic or genetic-environmental interactions between races. Therefore, further research is needed to elucidate this question.

In summary, this meta-analysis study did not show any association between ASPN polymorphisms and OA susceptibility in Asians, Europeans or Americans. Thus, our results do not support the common viewpoint that the ASPN polymorphisms (except for D17) constitute risk factors for OA susceptibility in Asians, Europeans or Americans. We observed that the D17 alleles of ASPN increased the risk for development of OA if the considered sample size is greater than 1,000. Because of large heterogeneity in the studies included in this meta-analysis, we consider that the effects of ASPN polymorphisms on OA development still require further confirmation by larger studies performed in homogeneous populations.

\section{Acknowledgements}

The authors thank the researchers of the original studies included in this meta-analysis.

\section{Funding}

The present study was supported by the youth research project of Shaanxi University of Chinese Medicine (2015QN05).

\section{Availability of data and materials}

All data generated or analyzed during this study are included in this published article.

\section{Author contributions}

RZ designed the study. JW, AY, JZ and RZ screened the literature. NS, XiangwenL, XinghuiL, QL, JL and XR extracted the data from the literature. JW, RZ and ZK conducted the meta-analysis and wrote the manuscript. RZ and JW submitted the study.

\section{Ethics approval and consent to participate}

Not applicable.

\section{Consent for publication}

Not applicable.

\section{Competing interests}

The authors declare that they have no competing interests.

\section{References}

1. González-Huerta NC, Borgonio-CuadraVM, Zenteno JC, Cortés-González S, Duarte-Salazar C and Miranda-Duarte A: D14 repeat polymorphism of the asporin gene is associated with primary osteoarthritis of the knee in a Mexican Mestizo population. Int J Rheum Dis 20: 1935-1941, 2015.
2. Song GG, Kim JH and Lee YH: A meta-analysis of the relationship between aspartic acid (D)-repeat polymorphisms in asporin and osteoarthritis susceptibility. Rheumatol Int 34: 785-792, 2014.

3. Jazayeri R, Qoreishi M,Hoseinzadeh H, Babanejad M, Bakhshi E, Najmabadi $\mathrm{H}$ and Jazayeri SM: Investigation of the asporin gene polymorphism as a risk factor for knee osteoarthritis in Iran. Am J Orthop (Belle Mead NJ) 42: 313-316, 2013.

4. Juchtmans N, Dhollander AA, Coudenys J, Audenaert EA, Pattyn C, Lambrecht S and Elewaut D: Distinct dysregulation of the small leucine-rich repeat protein family in osteoarthritic acetabular labrum compared to articular cartilage 67: 435-441, 2015.

5. Michael JW, Schlüter-Brust KU and Eysel P: The epidemiology, etiology, diagnosis, and treatment of osteoarthritis of the knee. Dtsch Arztebl Int 107: 152-162, 2010.

6. Xing D, Ma XL, Ma JX, Xu WG, Wang J, Yang Y, Chen Y, Ma BY and Zhu SW: Association between aspartic acid repeat polymorphism of the asporin gene and susceptibility to knee osteoarthritis: A genetic meta-analysis. Osteoarthritis Cartilage 21: 1700-1706, 2013.

7. Woolf AD and Pfleger B: Burden of major musculoskeletal conditions. Bull World Health Organ 81: 646-656, 2003.

8. Zhang Y and Jordan JM: Epidemiology of osteoarthritis. Clin Geriatr Med 26: 355-369, 2010

9. Haq SA and Davatchi F: Osteoarthritis of the knees in the COPCORD world. Int J Rheum Dis 14: 122-129, 2011.

10. Minafra L, Bravatà V, Saporito M, Cammarata FP, Forte GI, Caldarella S, D'Arienzo M, Gilardi MC, Messa C and Boniforti F: Genetic, clinical and radiographic signs in knee osteoarthritis susceptibility. Arthritis Res Ther 16: R91, 2014.

11. Fernandes MT, Fernandes KB, Marquez AS, Cólus IM, Souza MF, Santos JP and Poli-Frederico RC: Association of interleukin-6 gene polymorphism (rs1800796) with severity and functional status of osteoarthritis in elderly individuals. Cytokine 75: 316-320, 2015.

12. Rushton MD, Reynard LN, Young DA, Shepherd C, Aubourg G, Gee F, Darlay R, Deehan D, Cordell HJ and Loughlin J: Methylation quantitative trait locus analysis of osteoarthritis links epigenetics with genetic risk. Hum Mol Genet 24: 7432-7444, 2015.

13. Bijsterbosch J, Kloppenburg M, Reijnierse M, Rosendaal FR, Huizinga TW, Slagboom PE and Meulenbelt I: Association study of candidate genes for the progression of hand osteoarthritis. Osteoarthritis Cartilage 21: 565-569, 2013.

14. Nakamura T, Shi D, Tzetis M, Rodriguez-Lopez J, Miyamoto Y, Tsezou A, Gonzalez A, Jiang Q, Kamatani N, Loughlin J and Ikegawa S: Meta-analysis of association between the ASPN D-repeat and osteoarthritis. Hum Mol Genet 16: 1676-1681, 2007.

15. Valdes AM, Loughlin J, Oene MV, Chapman K, Surdulescu GL, Doherty $M$ and Spector TD: Sex and ethnic differences in the association of ASPN, CALM1, COL2A1, COMP, and FRZB with genetic susceptibility to osteoarthritis of the knee. Arthritis Rheum 56: 137-146, 2007.

16. Kou I, Nakajima M and Ikegawa S: Expression and regulation of the osteoarthritis-associated protein asporin. J Biol Chem 282: 32193-32199, 2007.

17. Neptune ER, Frischmeyer PA, Arking DE, Myers L, Bunton TE, Gayraud B, Ramirez F, Sakai LY and Dietz HC: Dysregulation of TGF-beta activation contributes to pathogenesis in Marfan syndrome. Nat Genet 33: 407-411, 2003.

18. Mustafa Z, Dowling B, Chapman K, Sinsheimer JS, Carr A and Loughlin J: Investigating the aspartic acid (D) repeat of asporin as a risk factor for osteoarthritis in a UK Caucasian population. Arthritis Rheum 52: 3502-3506, 2005.

19. Atif U, Philip A, Aponte J, Woldu EM, Brady S, Kraus VB, Jordan JM, Doherty M, Wilson AG, Moskowitz RW, et al: Absence of association of asporin polymorphisms and osteoarthritis susceptibility in US Caucasians. Osteoarthritis Cartilage 16: 1174-1177, 2008.

20. Min SK, Nakazato K, Ishigami H and Hiranuma K: Cartilage intermediate layer protein and Asporin Polymorphisms are independent risk factors of lumbar disc degeneration in male collegiate athletes. Cartilage 5: 37-42, 2014.

21. Thakkinstian A, McEvoy M, Minelli C, Gibson P, Hancox B, Duffy D, Thompson J, Hall I, Kaufman J, Leung TF, et al: Systematic review and meta-analysis of the association between $\{$ beta\}2-adrenoceptor polymorphisms and asthma: A HuGE review. Am J Epidemiol 162: 201-11, 2005. 
22. Qin X, Peng Q, Tang W, Lao X, Chen Z, Lai H, Deng Y, Mo C, Sui J, Wu J, et al: An updated meta-analysis on the association of MDM2 SNP309 polymorphism with colorectal cancer risk. PloS One 8: e76031, 2013.

23. Arellano-Pérez-Vertti RD, Argüello-Astorga JR, Cortéz-López ME, Zamarripa-Mottú JI, García-Salcedo JJ and Luna-Ceniceros DA: D-repeat polymorphism in the ASPN gene in knee osteoarthritis in females in Torreón, Coahuila. Acta Ortop Mex 28: 363-368, 2014.

24. Maris $P$, Blomme A, Palacios AP, Costanza B, Bellahcène $A$, Bianchi E, Gofflot S, Drion P, Trombino GE, Di Valentin E, et al: Asporin is a fibroblast-derived TGF- $\beta 1$ inhibitor and a tumor suppressor associated with good prognosis in breast cancer. PLoS Med 12: e1001871, 2015.

25. Shi D, Dai J, Zhu P, Qin J, Zhu L, Zhu H, Zhao B, Qiu X, Xu Z, Chen D, et al: Association of the $\mathrm{D}$ repeat polymorphism in the ASPN gene with developmental dysplasia of the hip: A case-control study in Han Chinese. Arthritis Res Ther 13: R27, 2011.

26. Duval E, Bigot N, Hervieu M, Kou I, Leclercq S, Galéra P, Boumediene $\mathrm{K}$ and Baugé $\mathrm{C}$ : Asporin expression is highly regulated in human chondrocytes. Mol Med 17: 816-823, 2011.

27. Kizawa H, Kou I, Iida A, Sudo A, Miyamoto Y, Fukuda A, Mabuchi A, Kotani A, Kawakami A, Yamamoto S, et al: An aspartic acid repeat polymorphism in asporin inhibits chondrogenesis and increases susceptibility to osteoarthritis. Nat Genet 37: 138-144, 2005.
28. Jiang Q, Shi D, Yi L, Ikegawa S, Wang Y, Nakamura T, Qiao D, Liu C and Dai J: Replication of the association of the aspartic acid repeat polymorphism in the asporin gene with knee-osteoarthritis susceptibility in Han Chinese. J Hum Genet 51: 1068-1072, 2006.

29. Kaliakatsos M, Tzetis M, Kanavakis E, Fytili P, Chouliaras G, Karachalios T, Malizos K and Tsezou A: Asporin and knee osteoarthritis in patients of Greek origin. Osteoarthritis Cartilage 14: 609-611, 2006

30. Song JH, Lee HS, Kim CJ, Cho YG, Park YG, Nam SW, Lee JY and Park WS: Aspartic acid repeat polymorphism of the asporin gene with susceptibility to osteoarthritis of the knee in a Korean population. Knee 15: 191-195, 2008.

31. Arellano RD, Hernández F, García-Sepúlveda CA, Velasco VM, Loera CR and Arguello JR: The D-repeat polymorphism in the ASPN gene and primary knee osteoarthritis in a Mexican mestizo population: A case-control study. J Orthop Sci 18: 826-831, 2013.

32. Rodriguez-Lopez J, Pombo-Suarez M, Liz M, Gomez-Reino JJ and Gonzalez A: Lack of association of a variable number of aspartic acid residues in the asporin gene with osteoarthritis susceptibility: Case-control studies in Spanish Caucasians. Arthritis Res Ther 8: R55, 2006.

This work is licensed under a Creative Commons Attribution-NonCommercial-NoDerivatives 4.0 International (CC BY-NC-ND 4.0) License. 